\title{
WARPED PRODUCTS AND CONFORMAL BOUNDARIES OF CAT(0)-SPACES
}

\author{
STEPHEN M. BUCKLEY AND SIMON L. KOKKENDORFF
}

\begin{abstract}
We discuss the conformal boundary of a warped product of two length spaces and provide a method to calculate this in terms of the individual conformal boundaries. This technique is then applied to produce CAT(0)-spaces with complicated conformal boundaries. Finally we prove that the conformal boundary of an Hadamard $n$-manifold is always simply connected for $n \geq 3$, thus providing a bound for the level of complication of the boundary of such a manifold.
\end{abstract}

Keywords: Length space, conformal boundary, ideal boundary, warped product, CAT(0)space, fundamental group. 2000 MSC: 53C20; 53C22; 53A30

\section{INTRODUCTION}

There are various notions of boundaries at infinity of a metric space. In this paper we will discuss and compare two of these.

The ideal boundary is a classical concept usually defined as a set of equivalence classes of paths converging to infinity. The precise definition will be given in the next section. Conformal boundaries are defined via conformal distortions of the metric space, and thus depend on the choice of distortion function. In certain classes of spaces, e.g. Gromov hyperbolic spaces, there is a range of canonical choices of distortions, which produce homeomorphic boundaries, c.f. [3, Chapter 3]. These turn out also to be homeomorphic to the ideal boundary with a canonically defined topology, see [5, Theorem 2.4]. In the class of CAT(0)-spaces however, there is no canonical choice of conformal distortion, and the ideal and conformal boundary will typically be different, when the space is not Gromov hyperbolic. This is the case e.g. when there are flat "sections" in the space extending to infinity.

One of the main purposes of this paper is to determine the conformal boundary of a warped product with warping function in a class of functions of distance to a base point. This we do in Section 3 after some preliminaries in Section 2. In Section 4, we use our warped product characterization to produce CAT(0)-spaces where the two types of boundary differ in interesting ways.

In particular, we prove the following result. Note that the ideal boundary of every Hadamard $n$-manifold is homeomorphic to $\mathbb{S}^{n-1}$ [4, II.8.11].

Theorem 1.1. For each $n>2$, there exists an Hadamard n-manifold whose conformal boundary is homeomorphic to a 1-point union of two $(n-1)$-spheres and an Hadamard $n$-manifold whose conformal boundary is homeomorphic to an $(n-2)$-disk.

Date: 2 September 2007.

The first author was partly supported by Enterprise Ireland and Science Foundation Ireland. The second author was partly supported by Enterprise Ireland and the Danish Research Agency. 
Finally in Section 5, we show that even though the conformal boundary of an Hadamard manifold can be quite complicated from a topological viewpoint, it is at least simply connected, when the dimension of the manifold is greater than 2 .

Theorem 1.2. Let $M$ be an Hadamard $n$-manifold, $n \geq 3$, then the conformal boundary $\partial_{\rho} M$ is simply connected.

\section{Preliminaries}

2.1. Length spaces and conformal distortions. We refer to [4] and [5] for more details on the concepts we briefly introduce below.

A length space is a metric space $(X, d)$, where the distance between two points is given as the infimum of lengths of rectifiable curves connecting points:

$$
d(p, q)=\inf _{\gamma \in \Gamma(p, q)} \mathfrak{L}(\gamma)
$$

where $\Gamma(p, q)$ denotes the set of rectifiable paths having $p$ and $q$ as endpoints. Given a continuous function $\rho: X \rightarrow(0, \infty)$, we can define the conformally distorted length metric:

$$
\sigma_{\rho}(p, q):=\inf _{\gamma \in \Gamma(p, q)} \int_{\gamma} \rho d s,
$$

see e.g. [3] for more details. One then defines the conformal boundary of $X$ with respect to the distortion $\rho$ as

$$
\partial_{\rho} X:=\bar{X}_{\rho} \backslash X
$$

where $\bar{X}_{\rho}$ denotes the metric completion of $X$ with respect to the length metric $\sigma_{\rho}$.

We will mainly focus attention on the case where the distortion is a function of the distance to a base point $o \in X$ :

$$
\rho(p)=g(d(o, p))
$$

and $g:[0, \infty) \rightarrow(0, \infty)$ is a continuous function with $g \in L^{1}([0, \infty))$. We use $\partial_{g} X$ as a short notation for the boundary with respect to such a conformal distortion. If $X$ has an infinitely long geodesic ray emanating from $o$, e.g. if $X$ is proper and unbounded, then we always have $\partial_{g} X \neq \emptyset$. See [5] for more details on conformal boundaries of this type.

The restriction to conformal distortions which are distance functions will simplify the analysis, but is not essential. In Theorem 1.2, proven in the final section, we also consider a slightly larger class of deformations. One easy observation, allowing for generalizations of the class of exact distance type deformations, is the following:

Proposition 2.1. Let $X$ be a length space with base point $o \in X, g:[0, \infty) \rightarrow(0, \infty)$ a continuous function and $\rho: X \rightarrow(0, \infty)$ a continuous function such that

$$
\frac{1}{C} g(d(o, p)) \leq \rho(p) \leq C g(d(o, p))
$$

for some $C \geq 1$ and all $p \in X$. Then $\partial_{\rho} X$ is homeomorphic, in fact bi-Lipschitz equivalent, to $\partial_{g} X$.

The proof is trivial and is left to the reader. 
2.2. Ideal Boundaries. A $K$-rough geodesic $\gamma: I \rightarrow X$ is a path, such that for all $s<t \in I$, we have

$$
d(\gamma(s), \gamma(t)) \geq \mathfrak{L}(\gamma([s, t]))-K
$$

for some $K \geq 0$. Here we do do not distinguish notationally between paths and their images. If (2.2) holds with $K=0, \gamma$ is called a geodesic. A length space is called geodesic if any two points can be joined by a geodesic. Any proper (i.e. where closed balls are compact) length space is geodesic by the Hopf-Rinow Theorem [4, I.3.7].

A $K$-rough ray $\gamma$ in $X$ is an infinitely long $K$-rough geodesic with one endpoint, i.e. when parametrized by arclength it can be defined on $[0, \infty)$. A ray is then a 0 -rough ray, that is, an infinitely long geodesic with one endpoint.

Two paths $\gamma_{1}, \gamma_{2}$ are said to be asymptotic if the Hausdorff distance between their images is finite $d_{\mathcal{H}}\left(\gamma_{1}, \gamma_{2}\right)<\infty$. This defines an equivalence relation $\sim$ on the set of paths. One easily checks that for two rough rays $\gamma_{1}, \gamma_{2}$ parametrized by arclength, we have $\gamma_{1} \sim \gamma_{2}$ if and only if

$$
\sup _{t>0} d\left(\gamma_{1}(t), \gamma_{2}(t)\right)<\infty
$$

We define the ideal boundary $\partial_{I} X$ to be the set of equivalence classes of (geodesic) rays. It will prove convenient to work with the slightly more general notion of a rough ideal boundary, $\partial_{R I} X$, defined to be the set of equivalence classes of rough rays. Clearly, we have $\partial_{I} X \subseteq \partial_{R I} X$. If $X$ is Gromov hyperbolic then we have $\partial_{I} X=\partial_{R I} X$, however it is possible to have a strict inclusion:

Example 2.3. In general for an Hadamard manifold $M^{n}$, i.e. a complete, simply connected Riemannian manifold of nonpositive curvature, it is well known that $\partial_{I} M$ is homeomorphic to $\mathbb{S}^{n-1}$, when equipped with a canonically defined topology, c.f. [4, II.8.11]. Even in the simplest case $M=\mathbb{R}^{2}$, we have $\partial_{I} \mathbb{R}^{2} \subsetneq \partial_{R I} \mathbb{R}^{2}$. Given a ray $\gamma$ (a half-line going to infinity) it is possible to construct a rough ray, which "zig-zags" around $\gamma$, in a way such that the two paths are not asymptotic according to the definition above.

2.3. CAT(0)-spaces. A geodesic triangle $T$ in a metric space $X$ is the union of three geodesics, $\gamma_{1} \in \Gamma(a, b), \gamma_{2} \in \Gamma(b, c), \gamma_{3} \in \Gamma(c, a)$. A geodesic triangle $T$ is said to satisfy the $\operatorname{CAT}(0)$-inequality if it is at least as slim as a comparison triangle with the same side lengths $\tilde{T} \subset \mathbb{R}^{2}$. See e.g. [4, II.1.1] for the precise definition. A CAT(0)-space is then a geodesic space in which all geodesic triangles satisfy the CAT(0)-condition.

2.4. Warped Products. We will define the warped product of two length spaces as in [1] and [2], see also [7]:

Let $B$ and $F$ be length spaces, and let $f: B \rightarrow(0, \infty)$ be a continuous function. Then define the following length structure on $B \times F$ :

$$
\mathfrak{L}_{f}(\gamma)=\int_{\gamma} \sqrt{v_{B}^{2}(t)+f^{2}\left(\gamma_{B}(t)\right) v_{F}(t)^{2}} d t
$$

where $\gamma=\left(\gamma_{B}, \gamma_{F}\right)$ and $v_{B}, v_{F}$ are the speeds of $\gamma_{B}, \gamma_{F}$ respectively, which are defined almost everywhere. We will write

$$
d_{f}(p, q)=\inf _{\gamma \in \Gamma(p, q)} \mathfrak{L}_{f}(\gamma)
$$

for the length metric induced from the length structure $\mathfrak{L}_{f}$. The warped product $B \times_{f} F$ is then $B \times F$ equipped with the metric $d_{f}$. 
We use standard terminology as for Riemannian warped products, c.f. [9]. $B$ is called the base and $F$ is called the fiber. Subsets of the form $B \times\{q\}, q \in F$, are called leaves, while the subsets $\{p\} \times F, p \in B$ are called fibers. A curve of the form $t \mapsto(\alpha(t), q)$ is called horizontal, while $t \mapsto(p, \beta(t))$ is called a vertical curve.

From the definition of the warped product metric, it is clear that the projection onto the base coordinate $\pi_{B}: B \times_{f} F \rightarrow B$ is 1-Lipschitz,

$$
d_{B}\left(\pi_{B}(p), \pi_{B}(q)\right) \leq d_{f}(p, q) .
$$

It is also evident that the leaves $B \times\{q\}, q \in F$ are all isometric to $B$. Furthermore, if $f: B \rightarrow(0, \infty)$ has a global minimum at $p \in B$, one easily checks that the fiber $\{p\} \times F$ is isometric to $F$ with metric scaled by $f(p)$.

Lemma 2.5. The identity map $B \times{ }_{f} F \rightarrow B \times F$ is a homeomorphism when $B \times F$ is equipped with the product topology. If $B$ and $F$ are complete, then so is $B \times_{f} F$. Thus if $B$ and $F$ are proper, so is $B \times{ }_{f} F$.

Proof. Given $p, q \in B \times F$, we have

$$
d_{f}(p, q) \leq f\left(\pi_{B}(p)\right) d_{F}\left(\pi_{F}(p), \pi_{F}(q)\right)+d_{B}\left(\pi_{B}(p), \pi_{B}(q)\right),
$$

by first moving in the fiber through $p$ and then in the leaf through $q$. This shows that the identity is continuous from $B \times F$ to $B \times{ }_{f} F$.

Now choose a ball $\mathcal{B} \subset B$ of radius $r$ around $\pi_{B}(p)$ such that $f(x) \geq \frac{1}{2} f\left(\pi_{B}(p)\right)$ for $x \in \mathcal{B}$. Given $\epsilon>0$, choose $0<\delta<\min \left\{\epsilon, f\left(\pi_{B}(p)\right) \frac{\epsilon}{2}, r\right\}$. Then if $d_{f}(p, q)<\delta<r$ we can ensure that for an almost minimizing curve $\gamma=\left(\gamma_{B}, \gamma_{F}\right)$ connecting $p$ and $q$, we have $\gamma_{B} \subset \mathcal{B}$, since $\mathfrak{L}_{B}\left(\gamma_{B}\right) \leq \mathfrak{L}_{f}(\gamma)$. Thus

$$
\mathfrak{L}_{f}(\gamma) \geq\left(\min _{\gamma} f\right) \mathfrak{L}_{F}\left(\gamma_{F}\right) \geq \frac{1}{2} f\left(\pi_{B}(p)\right) \mathfrak{L}_{F}\left(\gamma_{F}\right) \geq \frac{1}{2} f\left(\pi_{B}(p)\right) d_{F}\left(\pi_{F}(p), \pi_{F}(q)\right)
$$

and we conclude that $d_{f}(p, q) \geq \frac{1}{2} f\left(\pi_{B}(p)\right) d_{F}\left(\pi_{F}(p), \pi_{F}(q)\right)$, hence $d_{F}\left(\pi_{F}(p), \pi_{F}(q)\right)<\epsilon$ and $d_{B}\left(\pi_{B}(p), \pi_{B}(q)\right)<d_{f}(p, q)<\epsilon$, which shows that the identity $B \times{ }_{f} F \rightarrow B \times F$ is continuous.

Since the projection $\pi_{B}$ is 1-Lipschitz, we get a Cauchy sequence $\left\{\pi_{B}\left(p_{n}\right)\right\} \subset B$, when $\left\{p_{n}\right\}$ is Cauchy in $B \times_{f} F$. An easy variation of the argument given above shows that also $\left\{\pi_{F}\left(p_{n}\right)\right\} \subset F$ is Cauchy, and thus the $d_{f}$-Cauchy sequence $\left\{p_{n}\right\}$ is convergent in the product topology, hence also in $B \times{ }_{f} F$.

Finally, if $B$ and $F$ are both proper, hence complete, then $B \times_{f} F$ is complete. $B \times_{f} X$ is also locally compact since the product topology is, hence $B \times{ }_{f} F$ is proper by the HopfRinow Theorem, c.f. [4, I.3.7].

\section{BOUNDARIES OF WARPED PRODUCTS}

To simplify the analysis, we will from now on consider warping functions, which are functions of the distance to a point. We assume that a base point $o_{B} \in B$ is chosen, and for $p \in B$ introduce the notation $|p|:=d_{B}\left(o_{B}, p\right)$. For $f:[0, \infty) \rightarrow(0, \infty)$ a continuous function, we consider warping functions of the form

$$
f \circ|\cdot|: B \rightarrow(0, \infty)
$$

and also use the shorthand notation $B \times{ }_{f} F$ for warped products with this type of composite warping functions. The meaning should be clear from context. 
The restriction to warping functions of this type is not essential, and the results below could be generalized by considering warping functions, which are comparable (in the right way) to functions of distance. However the class of warping functions we consider, provides us with sufficient flexibility to construct the examples of CAT(0)-spaces, we are looking for.

Lemma 3.1. Let $f:[0, \infty) \rightarrow[1, \infty)$ be a homeomorphism satisfying exponential growth conditions of the form

$$
\begin{array}{ll}
\exists a>0 \forall t>0: & f(t+a) \geq 2 f(t), \\
\exists A>0 \forall t>0: & f(t+1) \leq A f(t),
\end{array}
$$

Then the rough ideal boundary of $X:=B \times_{f} F$ is given by the set equation

$$
\partial_{R I} X=\left(\partial_{R I} B \times F\right) \cup \partial_{R I} F
$$

Proof. First of all, since all leaves $B \times\{q\}$ are isometric to $B$, and likewise because the fiber $\left\{o_{B}\right\} \times F$ is isometric to $F$ it is clear that $\left(\partial_{R I} B \times F\right) \cup \partial_{R I} F \subseteq \partial_{R I} X$. So we need to show that any rough ray $\gamma=\left(\gamma_{B}, \gamma_{F}\right)$ in $X$ is asymptotic to either a horizontal or a vertical rough ray.

Assume that $\gamma=\left(\gamma_{B}, \gamma_{F}\right)$ is a rough ray parametrized by arclength, so $v_{F} \leq 1 / f\left(\left|\gamma_{B}\right|\right)$ a.e. We begin by proving a couple of useful estimates for $\gamma$. By assumption there exists $K \geq 0$ such that for all $t \geq 0$ :

$$
\begin{aligned}
t-K & \leq d_{f}\left(\left(o_{B}, \gamma_{F}(0)\right), \gamma(t)\right) \\
& \leq d_{f}\left(\left(o_{B}, \gamma_{F}(0)\right),\left(o_{B}, \gamma_{F}(t)\right)\right)+d_{f}\left(\left(o_{B}, \gamma_{F}(t)\right), \gamma(t)\right) \\
& \leq \mathfrak{L}_{F}\left(\gamma_{F}([0, t])\right)+\left|\gamma_{B}(t)\right| \\
& \leq \int_{0}^{t} \frac{1}{f\left(\left|\gamma_{B}(s)\right|\right)} d s+\left|\gamma_{B}(t)\right|
\end{aligned}
$$

as is seen by first moving in the fiber $\left\{o_{B}\right\} \times F$, then in the base, and applying the triangle inequality.

If $\gamma_{B}(t) \nrightarrow o_{B}$ for $t \rightarrow \infty$ then there is some $\epsilon>0$ such that $\left|\gamma_{B}(t)\right|>\epsilon$ for some arbitrarily large values of $t$. Since the speed of $\gamma_{B}$ is bounded by 1 , it follows that $I:=\left\{t \geq 0:\left|\gamma_{B}(t)\right|>\epsilon / 2\right\}$ has infinite Lebesgue measure, and so

$$
t-\int_{0}^{t} \frac{d s}{f\left(\left|\gamma_{B}(s)\right|\right)}=\int_{0}^{t}\left(1-\frac{1}{f\left(\left|\gamma_{B}(s)\right|\right)}\right) d s \geq \mu(I \cap[0, t])\left(1-\frac{1}{f(\epsilon / 2)}\right) \rightarrow \infty
$$

Assume that $\left|\gamma_{B}(t)\right|$ is bounded, hence $\gamma_{F}$ and thus $F$ must be unbounded. It immediately follows from (3.5) that $\gamma_{F}$ must be a rough ray, so that $\gamma$ is asymptotic to the vertical ray $t \mapsto\left(o_{B}, \gamma_{F}(t)\right)$. In fact we have $\lim _{t \rightarrow \infty} \gamma_{B}(t)=o_{B}$, since if this were false then (3.7) and (3.6) together would contradict the boundedness of $\left|\gamma_{B}(t)\right|$.

Suppose instead that $\left|\gamma_{B}(t)\right|$ is unbounded. Again combining (3.7) and (3.6), we see that

$$
\left|\gamma_{B}(t)\right| \geq t-K-\mathfrak{L}_{F}\left(\gamma_{F}([0, t])\right) \geq t-K-\int_{0}^{t} \frac{1}{f\left(\left|\gamma_{B}(s)\right|\right)} d s \rightarrow \infty
$$


Thus $I:=\left\{t:\left|\gamma_{B}(t)\right|<c_{1}\right\}$ has finite measure, while $J:=\left\{t:\left|\gamma_{B}(t)\right|>2 c_{1}\right\}$ has infinite Lebesgue measure. Thus:

$$
\begin{aligned}
\frac{1}{f\left(c_{1}\right)} t-\mathfrak{L}_{F}\left(\gamma_{F}([0, t])\right) & \geq \frac{1}{f\left(c_{1}\right)} t-\int_{0}^{t} \frac{d s}{f\left(\left|\gamma_{B}(s)\right|\right)} \\
& =\int_{0}^{t}\left(\frac{1}{f\left(c_{1}\right)}-\frac{1}{f\left(\left|\gamma_{B}(s)\right|\right)}\right) d s \\
& \geq \mu(J \cap[0, t])\left(\frac{1}{f\left(2 c_{1}\right)}-\frac{1}{f\left(c_{1}\right)}\right)-\mu(I \cap[0, t]) f(0) \rightarrow \infty .
\end{aligned}
$$

Since $f(t) \rightarrow \infty$, this last estimate and (3.5) together imply that $\left|\gamma_{B}(t)\right|-c_{2} t \rightarrow \infty$ for any $c_{2} \in[0,1)$. By a change of parameters, we deduce that $\mathfrak{L}_{F}\left(\gamma_{F}\right) \leq C \int_{0}^{\infty} \frac{1}{f\left(\left|\gamma_{B}(t)\right|\right)} d t$ is finite, and thus that $\left|\gamma_{B}(t)\right|>t-K_{2}$ for some $K_{2}>0$, i.e. $\gamma_{B}$ is a rough ray in $B$.

Since $\mathfrak{L}_{F}\left(\gamma_{F}\right)<\infty$, we see that $\gamma_{F}(t)$ is convergent to some $p \in F$. Thus $\gamma$ is asymptotic to the horizontal rough ray $t \mapsto\left(\gamma_{B}(t), p\right)$, since by moving vertically it follows that:

$$
\sup _{t>0} d\left(\gamma(t),\left(\gamma_{B}(t), p\right)\right) \leq \sup _{t>0} f\left(\left|\gamma_{B}(t)\right|\right) \int_{t}^{\infty} \frac{1}{f\left(\left|\gamma_{B}(s)\right|\right)} d s<\infty
$$

In fact, in view of (3.3) and the double inequality $t-K_{3} \leq\left|\gamma_{B}(t)\right| \leq t+K_{3}$ for some $K_{3}>0$ and all $t \geq 0$, the second inequality in (3.8) reduces to the inequality

$$
\sup _{t>0} f(t) \int_{t}^{\infty} \frac{d s}{f(s)}<\infty
$$

and this follows from (3.2).

Let us return to the conformal boundary $\partial_{g} X$, defined with respect to a base point $o \in X$ and a continuous function $g:[0, \infty) \rightarrow(0, \infty)$, with $g \in L^{1}([0, \infty))$. We will call $g k$-quasidecreasing if

for all $0 \leq s \leq t$ and some $k \geq 1$.

$$
g(t) \leq k g(s)
$$

There is a map

$$
J_{X}: \partial_{R I} X \rightarrow \partial_{g} X
$$

defined by choosing sequences going to infinity along each rough ray, e.g. $x_{n}=\gamma(n), n \in$ $\mathbb{N}$. We will need the following result:

Lemma 3.9. If $X$ is a proper unbounded length space and $g:[0, \infty) \rightarrow(0, \infty)$ a quasidecreasing distortion function in $L^{1}([0, \infty))$, then $J_{X}: \partial_{R I} X \rightarrow \partial_{g} X$ is well defined and surjective. In fact $J_{X}\left(\partial_{I} X\right)=\partial_{g} X$.

Proof. First of all, we need to see that given a rough ray $\gamma, \gamma\left(t_{n}\right)$ will define an element in $\partial_{g} X$, for $t_{n}$ a parameter sequence converging to $\infty$. Since $\gamma$ is a rough ray we have $d(o, \gamma(t)) \geq t-K$ for some fixed $K>0$. Thus $g(d(o, \gamma(t))) \leq k g(t-K)$, if $g$ is $k$-quasidecreasing. Since $g \in L^{1}([0, \infty))$ it follows that the $\sigma_{g}$-length of $\gamma$ is finite, and thus that $\gamma\left(t_{n}\right)$ will define a $\sigma_{g}$-Cauchy sequence for any $t_{n} \rightarrow \infty$; here $\sigma_{g}$ is the conformally distorted metric.

That $J_{X}$ is well defined, i.e. that it does not depend on the choice of representative of an equivalence class of rough rays nor on the choice of sequence $\left\{\gamma\left(t_{n}\right)\right\}$ for $t_{n} \rightarrow \infty$, follows also from $g \in L^{1}([0, \infty))$ and so $g(t) \rightarrow 0$ for $t \rightarrow \infty$. So for any two sequences 
$x=\left\{x_{n}\right\}, y=\left\{y_{n}\right\}$ chosen along equivalent rays, we have $\sigma_{g}\left(x_{n}, x_{m}\right) \rightarrow 0, \sigma_{g}\left(y_{n}, y_{m}\right) \rightarrow$ 0 and $\sigma_{g}\left(x_{n}, y_{m}\right) \rightarrow 0$ for $n, m \rightarrow \infty$. Thus $x$ and $y$ will define equivalent $\sigma_{g}$-Cauchy sequences converging to some point in $\partial_{g} X$.

That $J_{X}$ is surjective, in fact already as a map from $\partial_{I} X \subseteq \partial_{R I} X$ follows from Theorem 2.1 and Theorem 2.2 in [5]. In short an argument goes as follows: given a $\sigma_{g}$-Cauchy sequence $\left\{x_{n}\right\} \subset X$ converging to a point in $\partial_{g} X$, we must have $d\left(o, x_{n}\right) \rightarrow \infty$, for any fixed point $o \in X$. In the proof of Theorem 2.2(b) a geodesic ray $\gamma$ with $\gamma(0)=o$ is constructed such that $d\left(\gamma, y_{n}\right) \leq 1$, where $y=\left\{y_{n}\right\}$ is a subsequence of $\left\{x_{n}\right\}$. Clearly $J_{X}(\gamma)$ is then equivalent to $y$, since $g(t) \rightarrow 0$ for $t \rightarrow \infty$. Furthermore a subsequence of a $\sigma_{g}$-Cauchy sequence is equivalent to the original sequence.

Now we fix also a base point $o_{F} \in F$ and thus a base point $o_{X}:=\left(o_{B}, o_{F}\right) \in X=$ $B \times_{f} F$. We use the notation $|p|_{B}:=d_{B}\left(o_{B}, p\right),|q|_{F}:=d_{F}\left(o_{F}, q\right),|r|_{X}:=d_{X}\left(o_{X}, r\right)$ for points $p \in B, q \in F, r \in X . \sigma_{B}, \sigma_{F}$ and $\sigma_{X}$ will denote the conformally distorted metrics of $B, F$ and $X$ with respect to the chosen base points and a fixed distortion function $g$.

Theorem 3.10. Let $B$ and $F$ be pointed, proper length spaces, with $B$ unbounded. Let $f:[0, \infty) \rightarrow[1, \infty)$ be a warping function satisfying the requirements of Lemma 3.1 and let $g:[0, \infty) \rightarrow(0, \infty)$ be a distortion function as in Lemma 3.9 satisfying also:

$$
g(a+b) \geq k_{1} g(a) g(b) \text { and } f(t) g(t) \geq k_{2},
$$

for some positive constants $k_{1}, k_{2}$ and all $a, b, t \geq 0$. Then the conformal boundary $\partial_{g} X$ of the warped product $X=B \times_{\tilde{f}} F$, where $\tilde{f}=f \circ|\cdot|_{B}$, is homeomorphic to the gluing of $\partial_{g} B \times \bar{F}$ onto $\partial_{g} F$ along $\partial_{g} B \times \partial_{g} F$, using the projection map. If $\partial_{g} F=\emptyset$, the gluing is simply $\partial_{g} B \times F$.

Proof. For each leaf the embedding $B \hookrightarrow B \times\{q\} \subseteq X$ is isometric, and $|(p, q)|_{X} \geq|p|_{B}$, hence $g\left(|(p, q)|_{X}\right) \leq k_{0} g\left(|p|_{B}\right)$. Thus clearly any $\sigma_{B}$-Cauchy sequence $\left\{p_{n}\right\} \subset B$ is also a $\sigma_{X^{-}}$Cauchy sequence in the leaf $B \times\{q\}$. Hence we have a map

$$
\psi_{B}: \partial_{g} B \times F \rightarrow \partial_{g} X
$$

Likewise the embedding of the standard fiber $F \hookrightarrow\left\{o_{B}\right\} \times F \subset X$ is isometric, with $\left|\left(o_{B}, q\right)\right|_{X}=|q|_{F}$, so we also have a map

$$
\psi_{F}: \partial_{g} F \rightarrow \partial_{g} X
$$

Now define a map $\psi: \partial_{g} B \times \bar{F} \rightarrow \partial_{g} X$ by

$$
\psi((x, y))= \begin{cases}\psi_{B}((x, y)) & (x, y) \in \partial_{g} B \times F \\ \psi_{F}(y) & (x, y) \in \partial_{g} B \times \partial_{g} F\end{cases}
$$

Another way of getting the gluing of $\partial_{g} B \times \bar{F}$ and $\partial_{g} F$ is by considering the quotient $\left(\partial_{g} B \times \bar{F}\right) / \sim$, where $\partial_{g} B \times\{y\}$ is collapsed to a point for $y \in \partial_{g} F$. Since $\psi$ is constant on equivalence classes we may consider it as a map $\psi:\left(\partial_{g} B \times \bar{F}\right) / \sim \rightarrow \partial_{g} X$.

Equip $\partial_{g} B \times \bar{F}$ with the product topology induced by the conformally distorted metrics $\sigma_{B}, \sigma_{F}$ and give $\left(\partial_{g} B \times \bar{F}\right) / \sim$ the quotient topology. We will show that $\psi$ is a homeomorphism.

By Lemma 3.9 the conformal distortion map $J_{X}: \partial_{R I} X \rightarrow \partial_{g} X$ is surjective. Using the description in Lemma 3.1, it follows that $\psi$ is surjective. In fact, if $x \in \partial_{g} X$ and we choose a rough ray $\gamma$ such that $J_{X}(\gamma)=x$, then either $\gamma$ is asymptotic to a horizontal ray 
$\left(\gamma_{B}, q\right)$ and $x$ is in the image $\psi_{B}\left(\partial_{g} B \times F\right)$, or $\gamma$ is asymptotic to a vertical ray $\left(o_{B}, \gamma_{F}\right)$ and $x$ is in the image $\psi_{F}\left(\partial_{g} F\right)$.

To prove that $\psi$ is injective, we first need some estimates. Let $\gamma=(\alpha, \beta):\left[t_{0}, t_{1}\right] \rightarrow X$ be a path in $X$. By first moving in the fiber $\left\{o_{B}\right\} \times F$ and then horizontally in a leaf, we have a triangle with side lengths $|\beta(s)|_{F},|\alpha(s)|_{B},|(\alpha(s), \beta(s))|_{X}$, so by the triangle inequality:

$$
|(\alpha(s), \beta(s))|_{X} \leq|\alpha(s)|_{B}+|\beta(s)|_{F}
$$

so, using that $g$ is quasidecreasing and condition (3.11), we have

$$
g\left(|(\alpha(s), \beta(s))|_{X}\right) \geq k_{0} g\left(|\alpha(s)|_{B}+|\beta(s)|_{F}\right) \geq k_{0} k_{1} g\left(|\beta(s)|_{F}\right) g\left(|\alpha(s)|_{B}\right)
$$

Then

$$
\begin{aligned}
\int_{\gamma} g\left(|\gamma(s)|_{X}\right) \sqrt{v_{\alpha}^{2}+f^{2}\left(|\alpha(s)|_{B}\right) v_{\beta}^{2}} d s & \\
& \geq \int_{\gamma} k_{0} k_{1} g\left(|\beta(s)|_{F}\right) g\left(|\alpha(s)|_{B}\right) f\left(|\alpha(s)|_{B}\right) v_{\beta} d s
\end{aligned}
$$

By assumption there is a positive constant $k_{2}$ such that $g(t) f(t) \geq k_{2}$ for all $t>0$. Hence

$$
\mathfrak{L}_{\sigma_{X}}(\gamma) \geq k_{0} k_{1} k_{2} \mathfrak{L}_{\sigma_{F}}(\beta)
$$

so we conclude that

$$
\sigma_{X}\left(\left(p_{1}, q_{1}\right),\left(p_{2}, q_{2}\right)\right) \geq K_{0} \sigma_{F}\left(q_{1}, q_{2}\right)
$$

where $K_{0}=k_{0} k_{1} k_{2}$. A similar crude analysis shows that

$$
\mathfrak{L}_{\sigma_{X}}(\gamma) \geq K_{1} \min _{t_{0} \leq t \leq t_{1}}\left(g\left(|\beta(t)|_{F}\right)\right) \mathfrak{L}_{\sigma_{B}}(\alpha)
$$

where $K_{1}=k_{0} k_{1}$, and we conclude from (3.14) that

$$
\begin{aligned}
\epsilon:=\sigma_{X}\left(\left(p_{1}, q_{1}\right),\right. & \left.\left(p_{2}, q_{2}\right)\right) \\
& \geq K_{1} \min \left\{g(t): t \in\left[\left|q_{1}\right|_{F}-\epsilon / K_{0},\left|q_{2}\right|_{F}+\epsilon / K_{0}\right]\right\} \sigma_{B}\left(p_{1}, p_{2}\right),
\end{aligned}
$$

assuming that $\left|q_{1}\right|_{F} \leq\left|q_{2}\right|_{F}$. By continuity (3.15) extends to $\partial_{g} X$, i.e. to Cauchy sequences of the form $(x, y)$, for $x \in \bar{B}, y \in \bar{F}$. The estimate (3.17) extends to Cauchy sequences of the form $(x, q), x \in \partial_{g} B, q \in F$, i.e. to the image $\psi\left(\partial_{g} \times F\right)$.

We then easily deduce that $\psi$ is injective on $\left(\partial_{g} B \times \bar{F}\right) / \sim$. Because if $\psi\left(\left(x_{1}, y_{1}\right)\right)=$ $\psi\left(\left(x_{2}, y_{2}\right)\right)$ we must have $y_{1}=y_{2}:=y \in \bar{F}$ by (3.15), and for $y \in F$ we see from (3.16) that $\psi\left(\left(x_{1}, y\right)\right)=\psi\left(\left(x_{2}, y\right)\right)$ if and only if $x_{1}=x_{2}$.

Using (3.15) and (3.17) we see that $\psi^{-1}$ is continuous on the horizontal sequences $\psi\left(\partial_{g} \times F\right)$. Continuity of $\psi^{-1}$, in the quotient topology, at a vertical sequence $\psi_{F}\left(\partial_{g} F\right)$ follows directly from (3.15).

A similar analysis shows that $\psi$ is continuous. However this also follows from the fact that the involved spaces are compact and Hausdorff. 


\section{Exotic Boundaries of CAT(0)-Spaces}

In this section we apply the previous results to give examples of boundaries of CAT(0)spaces. That the ideal and conformal boundaries typically differ in this category is illustrated by the simplest example: $\partial_{I} \mathbb{R}^{n}$ is homeomorphic to $\mathbb{S}^{n-1}$, but $\partial_{g} \mathbb{R}^{n}$ is always a single point for $n \geq 2$, and any quasidecreasing distortion function $g \in L^{1}([0, \infty))$.

Flat "sections" extending to infinity will always be collapsed in the conformal boundary. In this respect the conformal boundary is in a way dual to the so-called Tits boundary, c.f. [4]. However negatively curved pieces can also be collapsed, if one chooses a conformal scaling function with too fast decay.

The following theorem is the main result in [1]:

Theorem 4.1 (Alexander \& Bishop). If $B$ and $F$ are complete CAT(0) spaces and $f: B \rightarrow(0, \infty)$ is convex, then $B \times{ }_{f} F$ is $C A T(0)$.

If we furthermore require that the function $f:[0, \infty) \rightarrow[1, \infty)$ in the construction of Theorem 3.10 is convex then, since distance functions are convex in CAT(0)-spaces [4, II.2.2], it follows easily that the composition $\left.(f \circ|\cdot|)\right|_{B}$ is convex and thus, by the above theorem of Alexander and Bishop, that $B \times_{f} F$ is $\operatorname{CAT}(0)$ when $B$ and $F$ are CAT(0)-spaces.

4.1. Examples. We give a few examples of complete, Riemannian CAT(0)-spaces

(i.e. Hadamard manifolds) with interesting boundary, calculated using Theorem 3.10. When $B$ is an Hadamard manifold, we get a smooth convex warping function satisfying the requirements of Lemma 3.1 by choosing $f(t)=\cosh (t)$.

If an Hadamard manifold $X^{n}$ has curvature bounded away from zero, $\kappa \leq \kappa_{0}<0$, and is thus Gromov hyperbolic, then the conformal boundary $\partial_{g} X$ is homeomorphic to the ideal boundary $\mathbb{S}^{n-1}$ when we choose the distortion $g$ to satisfy the so-called weak Floyd conditions

$$
\begin{gathered}
g(t) \leq C g(s), \quad \text { whenever } s, t \geq 0, s-1 \leq t \leq 2 s+1, \\
\quad \int_{0}^{\infty} g(t) d t \leq C g(0),
\end{gathered}
$$

for some fixed $C>0$, together with the decay condition $g(t) \geq K \exp \left(-\epsilon_{0} t\right)$ for some sufficiently small $\epsilon_{0}=\epsilon_{0}(\kappa)>0$; see Section 1.6 and Theorem 2.4 of [5]. Below we call any conformal distortion function $g$ that satisfies these three conditions an $\epsilon_{0}$-moderate distortion function. For any given $\epsilon_{0}>0$, there always exist $\epsilon_{0}$-moderate distortion functions that fulfill the requirements of Theorem 3.10: for instance, it suffices to choose $g(t)=\exp (-\epsilon t)$ for sufficiently small $\epsilon \in\left(0, \epsilon_{0}\right]$.

With these choices of $f$ and $g$ we have:

- If $B=\mathbb{R}$ and $F=\mathbb{R}^{n-1}$, with $n>2$, then $X$ is CAT(0) and so $\partial_{I} X$ is homeomorphic to $\mathbb{S}^{n-1}$. However $\partial_{g} X$ is homeomorphic to $\mathbb{S}^{n-1} \sqcup_{p} \mathbb{S}^{n-1}$, a 1-point union of two $(n-1)$-spheres. This follows since $\partial_{g} \mathbb{R}^{n-1}$ is a single point $\{p\}$ and $\overline{\mathbb{R}^{n-1}}$ is $\mathbb{S}^{n-1}$, while $\partial_{g} \mathbb{R}$ consists of two points.

- If $B=\mathbb{R}^{2}$ and $F=\mathbb{H}^{n-2}$ is hyperbolic $n$-space, $n>2$, then $X$ is CAT(0) and so $\partial_{I} X$ is homeomorphic to $\mathbb{S}^{n-1}$. However $\partial_{g} X=\mathbb{B}^{n-2}$, the closed unit ball.

Note that the above pair of examples already prove Theorem 1.1. It is also clear that if we iterate this procedure with some of the ingredients described above, it is possible 
to construct disturbingly complicated conformal boundaries, even when $\partial_{I} X$ is a sphere of codimension 1 .

We also note that while $X \cup \partial_{I} X$ is contractible, being homeomorphic to a closed unit ball in Euclidean space, it is easy to construct examples where $\bar{X}$ is not contractible. This is the case in the first example above where $\partial_{g} X$ is homeomorphic to $\mathbb{S}^{n-1} \sqcup_{p} \mathbb{S}^{n-1}$.

Let us also give some non-Riemannian examples of CAT(0)-spaces. Here we can take e.g. $f(t)=\cosh (t)$ or $f(t)=\exp (t)$. Again $g$ is some appropriate distortion function and $X=B \times{ }_{f} F$.

- Let $B=\mathbb{R}$ and let $F$ be a tree $T$. Then $X$ is CAT(0), with $\partial_{g} X$ the doubling of $T$ along $\partial_{g} T$. In this example the ideal and conformal boundaries are the same.

- Let $B=\mathbb{R}^{2}$ and let $F$ be a tree $T$. Then $X$ is CAT(0) and $\partial_{g} X$ is simply the closure of the tree $\bar{T}=T \cup \partial_{g} T$.

- Let $B$ be a tree with finitely many branchings, so that $\partial_{g} B$ is finite. Then $\partial_{g} X$ is finitely many copies of $\bar{F}$ glued along $\partial_{g} F$. If $F$ is $\operatorname{CAT}(0)$, then so is $X$.

\section{Simply CONNECTED CONFORMAL BOUNDARIES}

In this section we discuss further the topological properties of conformal boundaries of Hadamard manifolds. In particular we establish that the conformal boundary of an Hadamard manifold of dimension at least 3 is simply connected. Thus, even though we have seen that conformal boundaries of Hadamard manifolds can be quite complicated from a topological viewpoint, this provides a bound for the "level of complication".

We will need the concept of a quotient (pseudo)metric, see [4] p. 65, so let us discuss this. Given a metric space $(X, d)$ and an equivalence relation $\sim$ on $X$, there is a pseudometric $d^{\prime \prime}$ on $X$ given by the formula:

$$
d^{\prime \prime}(x, y)=\inf \left\{\sum_{i=1}^{m} d^{\prime}\left(x_{i-1}, x_{i}\right) \mid x_{0}=x, x_{m}=y\right\}, \quad x, y \in X .
$$

where

$$
d^{\prime}(u, v)= \begin{cases}0, & u \sim v \\ d(u, v), & \text { otherwise }\end{cases}
$$

and the infimum is taken over all finite chains of points in $X$ from $x$ to $y$. Since

$$
d^{\prime \prime}(x, y)=d^{\prime \prime}\left(x^{\prime}, y^{\prime}\right), \quad \text { whenever } x \sim x^{\prime}, y \sim y^{\prime},
$$

$d^{\prime \prime}$ induces a pseudometric $d_{\sim}$ on $X / \sim$, which we call the quotient pseudometric.

We call $\sim$ a metric equivalence relation if $d_{\sim}$ is a metric on $X / \sim$. In this section we let

$$
q: X \rightarrow X / \sim
$$

denote the quotient map.

For completeness we include the following useful characterization of metric equivalence relations on compact spaces.

Lemma 5.1. Suppose $(X, d)$ is a compact metric space and $\sim$ an equivalence relation on $X$. Then the following are equivalent:

(a) the relation $\sim$ is a metric equivalence relation;

(b) the quotient topology on $X_{\sim}$ is Hausdorff; 
(c) all equivalence classes in $X$ are closed and the quotient topology on $X / \sim$ equals the $d_{\sim}$ topology.

Proof. In this proof, let $\tau_{1}$ be the quotient topology, and $\tau_{2}$ be the $d_{\sim}$ topology on $X / \sim$. Since $q$ is a contraction, $\tau_{2}$ is always coarser than $\tau_{1}$.

It is easy to see that (a) implies (b) since if $d_{\sim}$ is a metric, then $\tau_{2}$ is Hausdorff and so $\tau_{1}$ is also Hausdorff.

We next prove that (b) implies (c), so suppose that $\tau_{1}$ is Hausdorff. Thus singletons in $X / \sim$ are closed and so equivalence classes in $X$ are closed. Every continuous bijection from a compact space to a Hausdorff space is a homeomorphism so, since $\left(X / \sim, \tau_{1}\right)$ is compact and $\left(X / \sim, \tau_{2}\right)$ is Hausdorff, it follows that $\tau_{1}=\tau_{2}$.

Lastly we prove that (c) implies (a). The closure of equivalence classes in $X$ is equivalent to $\tau_{1}$ being $T_{1}$. Assuming that $\tau_{1}$ is $T_{1}$ and that $\tau_{1}=\tau_{2}$, it follows that $d_{\sim}$ must be a metric since otherwise there would be distinct points $u, v \in X / \sim$ such that $d_{\sim}(u, v)=0$, and every $\tau_{2}$-neighborhood of $u$ would include $v$.

We note that there are equivalence relations on compact sets whose equivalence classes are closed but which fail to be metric equivalence relations. For instance, if $X$ is the Euclidean interval $[-1,1]$, and we define an equivalence relation whose equivalence classes are $\{x,-x\}, 0<x<1$, together with the singleton sets $\{0\},\{-1\}$, and $\{1\}$, then $d_{\sim}([-1],[1])=0$ even though -1 and 1 are inequivalent.

Note also that compactness is needed in the lemma above. Consider e.g. the complex plane, with the relation $0 \sim t i$ for all $t \geq 0$ and $1 \sim t i+1 /(t+1)$ for all $t \geq 0$ and no other nontrivial equivalence. Then $d_{\sim}([0],[1])=0$ but $[0] \neq[1]$, while the quotient topology is Hausdorff.

We will need the following result, which seems quite fundamental but which the authors were unable to find in the literature.

Proposition 5.2. If $X$ is a compact length space and $\sim$ a metric equivalence relation on $X$ with connected equivalence classes, then $X / \sim$ with the quotient metric is a compact length space and the natural map

$$
q_{*}: \pi_{1}(X) \rightarrow \pi_{1}(X / \sim)
$$

is surjective.

Proof. That in general $X / \sim$ is a compact length space, when $X$ is a compact length space and $\sim$ is a metric equivalence relation follows from Lemma 5.20 in [4], p.65.

In the following we assume that all curves are parametrized on the unit interval $I=$ $[0,1]$ and use standard notation from homotopy theory e.g. brackets $[\gamma]$ are used to denote homotopy classes.

We need to prove that $q_{*}$ is surjective, which amounts to showing that any homotopy class $[\gamma]$ in $\pi_{1}(X / \sim)$ can be represented by a lifted curve, $[\gamma]=[q \circ \omega]$, where $q: X \rightarrow$ $X / \sim$ is the quotient map. Since $X / \sim$ is a compact length space, it follows that any sufficiently short curve is homotopically trivial, c.f. [6] p. 215 . We thus assume that any curve of length less than $2 K>0$ in $X / \sim$ is homotopically trivial.

It is easy to see that any connected subset $C \subseteq X$ has the following property: Given any $x, y \in C$ and any $\epsilon>0$ there exists finitely many paths $\omega_{i}: I \rightarrow X, i \in\{1, \ldots, n\}$, such that $\omega_{1}(0)=x, \omega_{n}(1)=y, \omega_{i}(1)=\omega_{i+1}(0) \in C$ and $\mathfrak{L}\left(\omega_{i}\right) \leq \epsilon$. 
Thus $\omega=\omega_{1} * \cdots * \omega_{n} \in \Gamma(x, y)$ and if $C$ is an equivalence class of $\sim$ then $q \circ \omega$ consists of finitely many loops with $C \in X / \sim$ as basepoint. If $\epsilon<2 K$ then since $q$ is contracting (1-Lipschitz), $q \circ \omega$ will be homotopically trivial.

Let now a closed curve $\gamma: I \rightarrow X / \sim$ represent a homotopy class in $\pi_{1}(X / \sim)$. We may assume that $\gamma$ is rectifiable, since any free nontrivial homotopy class has a minimizing geodesic. Choose an $\epsilon \in(0, K)$ and parameter values $0=t_{1}<t_{2}<\cdots<$ $t_{n}=1$ in $I$, such that the length of each segment $\gamma\left(\left[t_{i}, t_{i+1}\right]\right)$ is less than $K-\epsilon$, hence $d_{\sim}\left(\gamma\left(t_{i}\right), \gamma\left(t_{i+1}\right)\right)<K-\epsilon$.

Consider a fixed $i \in\{1, \ldots, n-1\}$. By definition of $d_{\sim}$ we can find a finite chain of points $\left\{x_{1}, y_{1}, x_{2}, y_{2}, \ldots, x_{m}, y_{m}\right\} \subset X$ such that $d_{\sim}\left(\gamma\left(t_{i}\right), \gamma\left(t_{i+1}\right)\right)+\epsilon>\sum_{j=1}^{m} d\left(x_{j}, y_{j}\right)$, with $x_{1} \sim \gamma\left(t_{i}\right), y_{m} \sim \gamma\left(t_{i+1}\right)$ and $y_{j} \sim x_{j+1}$. Since equivalence classes are connected we have by the discussion above for each $j<m$ a path $\beta_{j} \in \Gamma\left(y_{j}, x_{j+1}\right)$ such that $q \circ \beta_{j}$ is homotopically trivial in $X / \sim$.

Consider then a continuous curve $\omega_{i}$ consisting of geodesic segments connecting each $x_{j}$ and $y_{j}$ as well as curve segments $\beta_{j}$ connecting $y_{j}$ to $x_{j+1}$ as above. That is, if $\alpha_{j} \in \Gamma\left(x_{j}, y_{j}\right)$ is a geodesic and $\beta_{j} \in \Gamma\left(y_{j}, x_{j+1}\right)$ is as above, we define

$$
\omega_{i}=\alpha_{1} * \beta_{1} * \alpha_{2} * \beta_{2} * \cdots * \beta_{m-1} * \alpha_{m} .
$$

Since the push forward of each $\beta_{j}$ curve is homotopically trivial and since $q$ is contracting it is now clear, that the push forward curve $q \circ \omega_{i}$ is homotopic to a curve $\tilde{\omega}_{i}$ connecting $\gamma\left(t_{i}\right)$ to $\gamma\left(t_{i+1}\right)$ with length

$$
\mathfrak{L}\left(\tilde{\omega}_{i}\right) \leq \sum_{j=1}^{m} d\left(x_{j}, y_{j}\right)<d_{\sim}\left(\gamma\left(t_{i}\right), \gamma\left(t_{i+1}\right)\right)+\epsilon .
$$

Thus the closed curve $\tilde{\omega}_{i} * \gamma_{\mid\left[t_{i}, t_{i+1}\right]}^{-1}$ has length less than $2 K$ and is thus homotopically trivial. It follows that $\tilde{\omega}_{i}$, and thus $q \circ \omega_{i}$, is homotopic to $\gamma_{\left[\mid t_{i}, t_{i+1}\right]}$.

For $i \in\{1, \ldots, n-1\}$ we get curves $\omega_{i}$ in $X$ as above, which we paste together to a continuous closed curve using curves $\zeta_{i} \in \Gamma\left(\omega_{i}(1), \omega_{i+1}(0)\right)$ (with indices counted modulo $n-1)$ such that $q \circ \zeta_{i}$ is homotopically trivial. Then $\gamma$ is homotopic to the curve built up of these segments,

$$
[\gamma]=\left[q_{*}\left(\omega_{1} * \zeta_{1} * \omega_{2} * \zeta_{2} * \cdots * \omega_{n-1} * \zeta_{n-1}\right)\right] .
$$

Note that in the generality of the proposition above, it is easy to construct examples, where some continuous curves in $X / \sim$ cannot be lifted to $X$; in contrary to the "usual" setting, where $q: X \rightarrow X / \sim$ is a fibration.

We are now ready to provide the main result of this section. Here we will allow more general conformal boundaries defined with respect to a continuous distortion $\rho: X \rightarrow(0, \infty)$ such that

$$
\rho \in C_{0}(X) \text { and } \rho \circ \gamma \in L^{1}([0, \infty))
$$

for any geodesic ray $\gamma \in \partial_{I} X$; here $C_{0}(X)$ denotes the continuous functions converging to zero at infinity. As we see from the proof of Lemma 3.9 this class of deformations includes the previously considered distance deformations $\rho=g(d(\cdot, o))$ with $g$ quasidecreasing.

Once again we will utilize the map $J_{X}: \partial_{I} X \rightarrow \partial_{\rho} X$, defined by taking sequences along rays. 
Lemma 5.4. Let $X$ be a proper $C A T(0)$-space and $\rho: X \rightarrow(0, \infty)$ satisfy (5.3), then $J_{X}: \partial_{I} X \rightarrow \partial_{\rho} X$ is well-defined, surjective and continuous.

Proof. The proof of well-definedness and surjectivity is similar to the proof of Lemma 3.9 , while continuity follows directly from the definition of the cone topology [4, II.8] and the fact that $d\left(o, x_{n}\right) \rightarrow \infty \Longrightarrow \rho\left(x_{n}\right) \rightarrow 0$ for any point $o \in X$, since $\rho \in C_{0}(X)$.

Corollary 5.5. The conformal boundary $\partial_{\rho} M$ of an Hadamard $n$-manifold, $n \geq 3$, is simply connected.

Proof. Since the map $J_{M}: \partial_{I} M \rightarrow \partial_{\rho} M$ is surjective, continuous and the considered spaces are compact, $\partial_{\rho} M$ has the quotient topology on $\partial_{I} M^{n} \cong \mathbb{S}^{n-1}$ induced by $J_{M}$.

We can extend $J_{M}$ to a map $M \cup \partial_{I} M \rightarrow \bar{M}$ by defining $J_{M}(x)=x$ for $x \in M$. It is a routine matter to check that this extension is continuous, with the cone topology on $M \cup \partial_{I} M$ which is homeomorphic to the closed unit ball in $\mathbb{R}^{n}$. We will then pull back the conformally distorted metric $\sigma_{\rho}$ to $M \cup \partial_{I} M$ using $J_{M}$, yielding the pseudometric $\tilde{\sigma}(x, y)=\sigma_{\rho}\left(J_{M}(x), J_{M}(y)\right)$, for $x, y \in M \cup \partial_{I} M$.

We only need to show that the fibers $J_{M}^{-1}(x)$ for $x \in \partial_{\rho} M$ are connected, allowing us to apply the previous proposition, since if we consider $\partial_{I} M$ as $\mathbb{S}^{n-1}$ equipped with its standard geodesic metric $d_{\mathbb{S}}$, then because the topology on $\partial_{\rho} M$ is Hausdorff, the quotient metric on $\partial_{\rho} M$ associated to this choice of metric on $\mathbb{S}^{n-1}$ becomes a length space metric ${ }^{1}$. If some fiber $F=J_{M}^{-1}(x) \subset M \cup \partial_{I} M$ is not connected, then since the fibers are closed $F$ can be decomposed into two disjoint closed sets $F=F_{1} \cup F_{2}$. Thus since $M \cup \partial_{I} M$ is compact, there is an open set $U$ such that $F_{1} \subset U$ and $\bar{U} \cap F_{2}=\emptyset$.

Thus any continuous curve joining a point $y_{1} \in F_{1}$ to a point $y_{2} \in F_{2}$ must intersect the compact set $\partial U \subset M \cup \partial_{I} M$. That $y_{1}$ and $y_{2}$ belong to the same equivalence class means that there exist curves $\gamma:[0,1] \rightarrow M \cup \partial_{I} M$ with $\gamma(0)=y_{1}, \gamma(1)=y_{2}$ and $\gamma((0,1)) \in M$ of arbitrarily short $\tilde{\sigma}$-length. Note that $\tilde{\sigma}=\sigma_{\rho}$ is a metric on $M$.

By compactness of $\partial U$ we have $\inf \left\{\tilde{\sigma}\left(y_{1}, y\right) \mid y \in \partial U\right\}=\delta>0$ and it follows that any curve $\gamma$ joining $y_{1}$ and $y_{2}$ as above will have $\tilde{\sigma}$-length at least $\delta$. Thus $y_{1}$ cannot be equivalent to $y_{2}$, which is a contradiction. Hence $J_{M}^{-1}(x)$ must be connected.

Notice that what we actually prove is: Suppose that we can put a topology on $X \cup \partial_{I} X$ such that $\partial_{I} X$ is homeomorphic to a length space, $X \cup \partial_{I} X$ is compact and $J_{X}: X \cup \partial_{I} X \rightarrow \bar{X}$ is continuous, then $J_{X *}: \pi_{1}\left(\partial_{I} X\right) \rightarrow \pi_{1}\left(\partial_{\rho} X\right)$ is surjective.

This statement of the result should apply to other spaces besides Hadamard manifolds and $\mathrm{CAT}(0)$-spaces.

\section{REFERENCES}

[1] S. Alexander and R. L. Bishop, Warped products of Hadamard spaces, Manuscripta Math. 96 (1998), no. 4, 487-505.

[2] S. Alexander and R. L. Bishop, Curvature bounds for warped products of metric spaces, Geom. Funct. Anal. 14 (2004), no. 6, 1143-1181.

[3] M. Bonk, J. Heinonen, and P. Koskela, Uniformizing Gromov hyperbolic spaces, Astérisque 270 (2001), 1-99.

[4] M. Bridson and A. Haefliger, Metric Spaces of Non-positive Curvature, Springer, 1999.

[5] S. Buckley and S. L. Kokkendorff, Comparing the Ideal and Floyd boundaries of a metric space, Trans. Amer. Math. Soc., to appear.

\footnotetext{
${ }^{1}$ Note that although this quotient metric will induce the same topology on $\partial_{\rho} M$ as the conformal metric $\sigma$, these metrics can be quite different in other respects!
} 
[6] I. Chavel, Riemannian Geometry: A modern introduction, Cambridge University Press, 1993.

[7] C. H. Chen, Warped products of metric spaces of curvature bounded from above, Trans. Amer. Math. Soc. 351 (1999), no. 12, 4727-4740.

[8] M. Coornaert, T. Delzant, and A. Papadopoulos, Géometrie et théories des groupes, Lecture Notes in Mathematics 1441, Springer, Berlin, 1990.

[9] B. O’Neill, Semi-Riemannian Geometry, Academic Press, 1983.

Department of Mathematics, National University of Ireland, Maynooth, Co. Kildare, IRELAND

E-mail address: sbuckley@maths.nuim.ie

Department of Mathematics, Technical University of Denmark, 2800 Kgs. Lyngby, DENMARK

E-mail address: S.L.Kokkendorff@mat.dtu.dk 